\title{
Die Cystische Fibrose im Wandel der Zeit
}

Prof. Dr. med. Nicolas Regamey, Luzern; Dr. med. Anne Mornand, Genf; Prof. Dr. med. Jürg Barben, St. Gallen

Am Beispiel der häufigsten Erbkrankheit beim Menschen, der Cystischen Fibrose (CF), lässt sich gut nachvollziehen, was die Schweiz für die steigenden Ausgaben im Gesundheitswesen erhält. In den letzten 30 Jahren haben medizinische Fortschritte Lebenserwartung und Lebensqualität von CF-Betroffenen drastisch verbessert, was nicht nur für die Betroffenen, sondern für die ganze Gesellschaft positive Auswirkungen hat.

Die Cystische Fibrose (CF), in Deutschland auch unter Mukoviszidose bekannt, ist eine der häufigsten Erbkrankheiten und bis heute leider unheilbar, wenn auch immer besser behandelbar. Etwa 5\% der Bevölkerung weisen auf einem Chromosom - meist ohne ihr Wissen - eine entsprechende Erbgutveränderung auf, erkranken jedoch nicht, da sie über ein zweites, gesundes Chromosom verfügen. Bei einem von etwa 3000 Neugeborenen haben jedoch beide Eltern ihr verändertes Chromosom weitergeben, so dass das Kind an CF erkrankt. Aktuell leben in der Schweiz etwa 1000 an CF erkrankte Menschen, heute sind bereits mehr Erwachsene als Kinder davon betroffen.

Bei CF-Betroffenen produzieren die Zellen aufgrund des Gendefekts zu zähflüssige Sekrete, was in erster Linie die Lungen- und die Verdauungsfunktion beeinträchtigt. Die CF zeigt sich in der Regel bereits im ersten Lebensjahr, da die Säuglinge u.a. unter Husten und Auswurf leiden, Bauchschmerzen haben und unzureichend an Gewicht zunehmen. Auch im weiteren Verlauf leiden die Betroffenen vor allem unter Entzündungen und Schädigungen des Lungengewebes sowie an der beeinträchtigten Funktion der Bauchspeicheldrüse, die auch zu einem gehäuften Auftreten von Diabetes führt.

\section{Die Lebenserwartung bei Cystischer Fibrose hat sich mehr als verdoppelt}

Lag die Lebenserwartung von CF-Betroffenen in den 80 er-Jahren noch bei knapp 20 Jahren, beträgt sie heutzutage fast 50 Jahre und steigt von Jahr zu Jahr weiter an. Kinderlungen-Spezialisten mussten vor 30 Jahren die Eltern eines Kindes mit CF mit der Tatsache kon- frontieren, dass es wahrscheinlich vor Erreichen des Erwachsenenalters sterben würde. Diese Kinderärzte haben dazumal Kinder mit CF öfters auf ihrem letzten Lebensabschnitt begleitet und an der Krankheit sterben sehen. Dies ist heutzutage zum Glück nur noch

Aktuell leben in der Schweiz etwa 1000 an CF erkrankte Menschen, heute sind bereits mehr Erwachsene als Kinder davon betroffen.

sehr selten der Fall, da die meisten CF-Betroffenen das Erwachsenenalter erreichen, viele davon mit einer fast normalen Lungenfunktion.

\section{Auch die Lebensqualität der Betroffe- nen und ihrer Familien hat sich stark verbessert}

Innerhalb dieser längeren Lebenszeit erfreuen sich CF-Betroffene heutzutage einer deutlich besseren Gesundheit, welche ihnen oft ermöglicht, sich gänzlich am sozialen und beruflichen Leben zu beteiligen. Menschen mit CF sind heute in fast allen Sparten von Berufen zu finden, sie treiben Sport und gründen oft auch Familien. Sie geben der Gesellschaft nicht nur menschlich und sozial, sondern auch finanziell einen Teil der in ihre Gesundheit investierten Kosten zurück.

Nicht nur die heute besser behandelbare Symptomatik, auch die verbesserten Therapieformen erhöhen die Lebensqualität der Betroffenen. Kinder müssen viel seltener die für sie und ihre Familien belastenden Spitalaufenthalte erdulden und können heute dank moderner Medizin ambulant behandelt werden. Intravenöse Antibiotikatherapien bedeuten heute nicht 
mehr zwingend einen zweiwöchigen Krankenhausaufenthalt, sondern oft eine Behandlung zuhause. So fehlen die Kinder bzw. jungen Erwachsenen weniger in der Schule oder bei der Arbeit. Im Weiteren konnten Therapien vereinfacht werden, wie zum Beispiel die Inhalationsbehandlungen, welche mehrmals täglich durchgeführt werden müssen. Dadurch macht es über den Tag und die Jahre betrachtet einen grossen Unterschied, dass eine Medikamenten-Verneblung, die vor 20 Jahren noch 15 Minuten in Anspruch nahm, heutzutage in fünf Minuten gemacht werden kann. Viele Inhalationsmedikamente sind heute als Pulver verfügbar.

\section{Gründe für die verbesserte Lebens- erwartung und Lebensqualität}

Ein vertieftes Wissen über die CF Erkrankung stellt den Grundpfeiler für verbesserte Behandlungsmöglichkeiten dar. Das Verständnis von pathophysiologischen $\mathrm{Zu}$ sammenhängen erlaubt die frühzeitige Entdeckung und Behandlung von Komplikationen der Krankheit. Heutzutage wird zum Beispiel eine CF-assoziierte $\mathrm{Zu}$ ckererkrankung früh gesucht und aggressiv mit Insulin therapiert. Auch können Medikamente wie die hypertone Kochsalzlösung oder die rekombinante DNAse präventiv gezielt angewendet werden, um Infektionen bzw. der Progression der Krankheit entgegen zu wirken.

\section{Menschen mit CF sind heute in fast allen Sparten von Berufen zu finden, sie treiben Sport und gründen oft auch Familien.}

Im Jahr 1989 identifizierten Forscher den Gendefekt, welcher der Krankheit zugrunde liegt (Defekt im CFTRGen, das für einen Salztransporter-Kanal auf der Membran von Epithelzellen kodiert). In der Folge konnten Therapien entwickelt werden, welche nicht nur die verheerenden Konsequenzen des defekten Salztransporter-Kanals bekämpfen, sondern die Krankheit an der Wurzel packen. Neuere Medikamente, sogenannte CFTRModulatoren können die Funktion des CFTR-Kanals verbessern und somit den Verlauf der Krankheit zumindest bei einigen Mutationen wesentlich beeinflussen bzw. abmildern. Neben solchen Entwicklungen neuer Medikamente sind für CF-Betroffene auch die
Fortschritte in der Transplantationsmedizin von Bedeutung, da eine Lungentransplantation das Leben von CF-Patienten bei guter Lebensqualität erheblich verlängern kann.

Dank der Einführung eines nationalen Screenings im Jahr 2011 kann heutzutage die CF bereits im Neugeborenenalter entdeckt werden, was Familien den oft langen und mühsamen, aber auch teuren Weg bis zur Diagnosestellung erspart und eine frühzeitige Behandlung der Krankheit mit verbesserter Prognose ermöglicht.

Last but not least hat auch die multidisziplinäre Vorgehensweise die Behandlung der CF stark verbessert. Ein Team von Pädiatern, Pneumologen, Pflegenden, Physiotherapeuten, Sozialarbeitern, Psychologen und Ernährungsberatern sorgt mit Vertretern anderer Disziplinen gemeinsam dafür, dass CF-Betroffene umfassend und ganzheitlich betreut werden, um ihre Lebenserwartung und -qualität zu verbessern. Den dafür anfallenden Kosten steht die deutlich längere und klar verbesserte Lebenszeit dieser Patientengruppe gegenüber, die nicht nur für die Betroffenen und ihr persönliches Umfeld, sondern auch für unsere Gesellschaft als Ganzes einen Gewinn darstellt.

\section{Kurzzusammenfassung - Nutzengewinn}

In den letzten 20 Jahren haben die Fortschritte der Medizin die Prognose von Menschen mit Cystischer Fibrose (CF) so verbessert, dass die Krankheit von einem rein pädiatrischen Thema zu einer Krankheit von Erwachsenen geworden ist. Früher waren Kinder mit CF schwer krank, erforderten rund um die Uhr Betreuung und Pflege und starben meistens vor dem Erreichen des Erwachsenenalters; heutzutage sind in der Schweiz bereits mehr als die Hälfte der CF-Betroffenen Erwachsene, und die Lebensqualität dieser Erwachsenen ist deutlich besser geworden. Neu gibt es auch die Möglichkeit einer Organtransplantation, was das Leben um viele Jahre mit einer guten Lebensqualität weiter verlängern kann. Erwachsene CF-Betroffene können in der Regel einen Beruf ausüben und nehmen am gesellschaftlichen Leben teil. Die einzelnen betroffenen Menschen, ihre Familien und ihr weiteres Umfeld sind sehr dankbar für diesen Fortschritt, der zwar einiges kostet, aber der Gesellschaft auch einen erheblichen Nutzen bringt! 